\title{
MALNUTRITION POINT-PREVALENCE FROM 2012 TO 2019 AND ASSOCIATED HEALTH-OUTCOMES IN ADULT PATIENTS IN RURAL HOSPITALS
}

\author{
E. Lopez ${ }^{1}$, M. Banbury ${ }^{2,3}$, E. Isenring ${ }^{4}$, S. Marshall ${ }^{5}$
}

\begin{abstract}
Background: Malnutrition negatively impacts hospitalised patients and the healthcare system. Objectives: 1) report pointprevalence of hospital malnutrition from 2012 to 2019; and 2) determine if there was an association between nutrition status and health-related outcomes. Design: Point-prevalence of malnutrition was determined by three $(2012,2014$, and 2019) cross-sectional studies. Health-related outcomes, assessed by a prospective cohort study in 2014, were length of stay, in-hospital mortality, hospital readmission, infection, falls, fractures, and pressure wounds. Setting: three Australian rural hospitals. Participants: Adult inpatients. Measurements: Nutrition status was assessed with the Subjective Global Assessment (SGA) tool. Results: Malnutrition point prevalence was 39\% in $2012(n=62), 48 \%$ in $2014(n=128)$, and 28\% in $2019(n=96)$; where the prevalence in 2019 was significantly lower than in 2014 ( $\mathrm{p}<0.017$ ). The 2019 (median age 70 years) sample was younger than the 2012 (median age 80 years) and 2014 (median age 78 years) samples $(\mathrm{p}<0.05)$. Mortality and falls rate were higher in the severely malnourished participants $(\mathrm{p}=<0.05)$; and severe malnutrition may predict mortality (Adjusted OR: 3.47 (95\%CI: 0.94, 12.78] p=0.061). Conclusions: Nutrition status did not predict other health-related outcomes. The rate of malnutrition in rural hospitals was consistently high and may increase the risk of in-hospital mortality.
\end{abstract}

Key words: Malnutrition, hospitals, nutrition assessment, subjective global assessment, mortality.

\section{Introduction}

Protein-energy malnutrition (herein referred to as 'malnutrition') negatively impacts the patient and healthcare system alike $(2,3)$, a major concern as the prevalence has been reported internationally at $30-50 \%$ across inpatient and residential settings, and 1-25\% across community settings (4-8). Malnutrition is the unintended loss of lean mass (muscle, immune and blood cells, viscera), with or without fat loss, due to inadequate intake, uptake, and/or utilisation of protein and energy to meet requirements $(4,9)$. Older adults are at greater risk of malnutrition due to their susceptibility of aetiological factors including psychological, socio-economic, and physiological changes and an overall increase in multi-

1. MNutrDietPrac, Accredited Practising Dietitian, Faculty of Health Sciences and Medicine, Bond University, Australia; 2. BSc (Hons) Applied Human Nutrition \& Dietetics. Accredited Practising Dietitian. Northern NSW Local Health District; 3. Faculty of Health Sciences and Medicine, Bond University, Australia; 4. PhD. Advanced Accredited Practising Dietitian. Bond University Nutrition \& Dietetics Research Group, Faculty of Health Sciences and Medicine, Bond University, Australia; 5. BNutr\&Diet (Hons), PhD. Accredited Practising Dietitian. Bond University Nutrition \& Dietetics Research Group, Faculty of Health Sciences and Medicine, Bond University, Australia

Corresponding Author: Skye Marshall, Nutrition \& Dietetics, Bond University, Robina, Queensland, 4226, Australia. Email: skye_marshall@bond.edu.au; Phone: 0755953337. morbidities and polypharmacy (9). A consequence of malnutrition is further morbidity, requiring increased healthcare resources including but not limited to hospital beds, multidisciplinary staff, and pharmaceutical and nutritional medicine (10). In particular, malnutrition increases risk of infection, pressure ulcers, poor wound healing, decreased response to medical treatment and pharmaceuticals, decreased respiratory function, decreased muscle repair, and overall functional impairment; leading to decreased quality of life and increased risk of mortality $(3,6,11)$.

While several large-scale studies have reported the prevalence and outcomes of hospital malnutrition, the rural context requires specific examination as populations in rural areas are ageing more rapidly than in urban areas $(11,15-17)$. Rural areas face increased challenges in providing health and aged care due to the higher cost of establishing and delivering services, the limited availability of and access to health professionals, and less availability of informal care networks (12-14). Not only is access to health care more limited in rural areas, rural-dwelling older adults are also more in need of health and aged care services. A recent meta-analysis and meta-regression of international data found the prevalence of malnutrition in rural-dwelling older adults living at home was double that of urban-dwelling older 
adults (5). Therefore, the prevalence and health-related outcomes of malnutrition in rural hospitals is of interest, so that policies may appropriately support patients in the continuum of care from hospital to home or residential care.

\section{Research aims}

In adult patients admitted to three rural hospitals in Australia, the aims of this study were to: 1 ) report pointprevalence of malnutrition from 2012 to 2019; and 2) determine if there was an association between nutrition status and health-related outcomes.

\section{Materials and Methods}

\section{Study design}

The point-prevalence of malnutrition was assessed using three cross-sectional studies conducted in 2012, 2014, and 2019. The association between malnutrition and health-related outcomes was evaluated using a prospective observational study in 2014. Participants gave their verbal consent to participate in the study. The project was approved by the Human Research Ethics Committees in April 2018 (QA249) as a quality assurance project. This study has been reported according to the STROBE Statement for cohort studies (15) and was retrospectively registered with ANZCTR (ACTRN12619000342112) (19).

\section{Setting and sample}

All three hospitals within a rural government-funded local health district in northern New South Wales, Australia, were conveniently sampled in 2012. Reflecting the staffing resources available for each cross-sectional study, the medical, surgical, general (not diagnosis or treatment specific), and/or rehabilitation wards were sampled (Table 1).

Table 1

Rural hospitals and wards sampled by the three crosssectional studies

\begin{tabular}{lll}
\hline Sample & Hospital & Wards included \\
\hline 2012 & Hospital 1 & Medical, surgical, rehabilitation \\
& Hospital 2 & \\
& Hospital 3 & \\
$2014^{*}$ & Hospital 1 & Medical, general, surgical, rehabilitation \\
& Hospital 2 & \\
& Hospital 3 & \\
2019 & Hospital 1 & Medical, general, surgical \\
\hline
\end{tabular}

* This sample was also that used in the prospective observational study.
The prospective cohort study was conducted using the 2014 sample due to availability of data and its larger sample size. Patients were eligible if they were 18 years or older and were admitted as inpatients to study sites during the recruitment phase of one to two weeks. No exclusion criteria were applied.

\section{Participant characteristics and potentially confounding variables}

Participant characteristics of age (years) and sex (male/female) were recorded for all participants. The 2014 sample were also described by comorbidities and medications. The number of active comorbidities were categorised into medical diagnostic groups: cancer, digestive, musculoskeletal, circulatory, respiratory, nervous, skin, reproductive, kidney, infectious, endocrine, injuries, ear, blood, and other. The number of medications were categorised into 24 drug classes based on the profile of medications recorded from the cohort (16).

\section{Outcomes}

Malnutrition was determined by the SGA tool which rates patients as $\mathrm{A}=$ well nourished, $\mathrm{B}=$ mild-moderate malnutrition, or $C=$ severe malnutrition $(17,18)$. The presence of malnutrition was the primary outcome to answer the first research question (point-prevalence) and the independent variable to answer the second research question (health-related outcomes).

The primary health-related outcome was length of hospital stay, defined by the number of days including the day of admission and discharge. Secondary healthrelated outcomes were in-hospital mortality (yes/no), hospital readmission (yes/no), in-hospital fall (yes/no), fall in subsequent hospital admissions (yes/no), pressure ulcer (yes/no), fracture acquired in hospital (yes/no), urinary tract or respiratory tract infection (yes/no). Health-related outcomes were measured from the day of hospital admission to three months post-discharge.

\section{Data Collection}

In 2012 and 2014, nutrition status was assessed by department dietitians over a 7-day period within each ward using the SGA. In 2019, nutrition status was assessed by one student-dietitian (EL) using the SGA over two weeks. Only the student dietitian in 2019 received training in correct SGA use, whereas department dietitians were expected to be competent in nutrition assessment due to years of experience. The SGA is comprised of two main components: medical and physical assessment. Changes in weight, dietary intake, gastrointestinal symptoms, and nutrition-related functional capacity were observed from a combination of patient records and patient interview for the medical 
Table 2

Age, sex, and malnutrition point-prevalence of the 2012, 2014, and 2019 participant samples

\begin{tabular}{|c|c|c|c|c|}
\hline Cohort year & $2012 n=62$ & $2014 n=128$ & $2019 n=96$ & p-value \\
\hline Age years (median (IQR)) & $80(60-85)$ & $78(65-86)$ & $70(58-82)$ & $<0.001,0.015^{*}$ \\
\hline $\operatorname{Sex}(\mathrm{n}(\%))$ & & & & $0.903+$ \\
\hline - Males & $28(45)$ & $60(47)$ & $51(53)$ & \\
\hline - Females & $34(54)$ & $68(53)$ & $45(47)$ & \\
\hline \multicolumn{5}{|l|}{ Malnourished (n (\%)) } \\
\hline -Total & $24(39)$ & $62(48)$ & $27(28)$ & $<0.001 \S$ \\
\hline -Males & $12(19)$ & $31,(24)$ & $13(14)$ & 0.794|| \\
\hline - Females & $12(19)$ & $31(24)$ & $14(15)$ & \\
\hline
\end{tabular}

component; while evidence of oedema, ascites, and loss of subcutaneous fat and muscle was assessed during a physical examination to inform the physical component. A patient who demonstrated negative changes to their oral intake and failed to meet their nutritional requirements with evidence of muscle and fat deterioration were classified as exhibiting a degree of malnutrition. Participant characteristics and the healthrelated outcomes were observed from the medical record.

\section{Data Analysis}

Data analysis was completed using IBM SPSS Statistics 25 [IBM Corp. Released 2017. IBM SPSS Statistics for Windows, Version 25.0. Armonk, NY: IBM Corp]. Descriptive statistics were used to summarise characteristics and outcome data. Continuous variables were considered non-normal if their skewness and kurtosis divided by their standard error exceeded +2 or -2; parametric variables were reported as mean (standard deviation) and non-parametric as median (IQR). Participants were considered "malnourished" if they were rated as SGA B or C, and "well-nourished" if rated SGA A. To determine if there was a significant difference in age, nutrition status, and sex between the samples, the Kruskal-Wallis $H$ Test was applied. If significance was observed, post-hoc testing using Mann-Whitney U was used for pairwise comparisons between cohorts. Here, the Bonferroni correction was applied at 0.05 level and adjusted for three groups; therefore, the cut-off value for significance for Mann-Whitney $U$ tests was at $p<0.017$. If significance was observed, post-hoc testing using MannWhitney U was used between pairs of cohorts. Post hoc power analysis using $G^{*}$ Power (version 3.1.9.2) was conducted on independent group means which showed significant difference. Extreme outliers (interquartile range rule with multiplier of 3 ) were removed from continuous variables. Binary logistic regression was used to determine the effect of the sample (2012, 2014, or 2019) on nutrition status, with age and sex as a confounding variables.

For the 2014 cohort, differences between nutrition status and outcomes were tested by the Mann-Whitney U Test or Chi-squared test. Associations with health-related outcomes were tested according to the level of severity, with patients considered as "malnourished" (SGA rating $B$ or $C$ ), and "severely malnourished" (SGA rating C). Multiple linear regression was used to determine the impact of nutrition status on LOS, and multiple logistic regression was used to determine the impact of nutrition status on secondary outcomes, accounting for participant characteristics which met assumptions for the model. Statistical significance was considered at the $p<0.05$ level two tailed unless otherwise indicated.

\section{Results}

\section{Participant characteristics}

A total of 286 participants were recruited; $n=62$ in 2012, $n=128$ in 2014, and $n=96$ in 2019 (Table 2). There was no difference in the sex ratio or the prevalence of malnutrition between the sexes in any sample. The 2019 sample (median age 70 years) was found to be significantly younger than the 2012 (median age 80 years) and 2014 (median age 78 years) samples ( $\mathrm{p}<$ $0.017)$. In the 2014 sample, a circulatory condition was the most common comorbidity experienced (72\%), followed by musculoskeletal and respiratory conditions (40\% and 38\% respectively) (Table 3). Cancer was more prevalent in malnourished $(33 \%)$ than well-nourished 
Table 3

Comorbidity and medication characteristics of 2014 sample

\begin{tabular}{|c|c|c|c|c|}
\hline Characteristic & Total sample $\left(\mathrm{n}=124^{\star}\right)$ & Well-nourished $(n=63)$ & Malnourished $(n=61)$ & p-valuet \\
\hline \multicolumn{5}{|l|}{ Comorbidities; n (\%) } \\
\hline - Cancer & $30(24)$ & $10(16)$ & $20(33)$ & 0.028 \\
\hline - Digestive & $38(31)$ & $22(35)$ & $16(26)$ & 0.294 \\
\hline - Musculoskeletal & $49(40)$ & $26(41)$ & $23(38)$ & 0.685 \\
\hline - Circulatory & $89(72)$ & $49(78)$ & $40(66)$ & 0.131 \\
\hline - Respiratory & $47(38)$ & $22(35)$ & $25(41)$ & 0.457 \\
\hline - Nervous & $8(7)$ & $4(6)$ & $4(7)$ & 0.962 \\
\hline - Skin & $13(11)$ & $8(13)$ & $5(8)$ & 0.413 \\
\hline - Reproductive & $11(9)$ & $5(8)$ & $6(10)$ & 0.710 \\
\hline - Kidney & $32(26)$ & $14(22)$ & $18(30)$ & 0.354 \\
\hline - Infectious & $24(19)$ & $12(19)$ & $12(20)$ & 0.930 \\
\hline - Endocrine & $45(36)$ & $25(40)$ & $20(33)$ & 0.425 \\
\hline - Injuries & $24(19)$ & $12(19)$ & $12(20)$ & 0.930 \\
\hline - Ear & $24(19)$ & $13(21)$ & $11(18)$ & 0.714 \\
\hline - Blood & $40(32)$ & $25(40)$ & $15(25)$ & 0.072 \\
\hline - Other & $38(31)$ & $16(25)$ & $22(36)$ & 0.198 \\
\hline Cumulative comorbidities & $4.1(1.8)$ & $4.2(1.6)$ & $4.1(1.9)$ & 0.770 \\
\hline
\end{tabular}

Medications class; $\mathrm{n}(\%)$

Total sample $(\mathrm{n}=128)$

Well-nourished $(n=66)$

Malnourished $(n=62)$

- Pain

$$
88(69)
$$

- Chemotherapy

- Cardiac

- Statin

- Anxiety / depression

- Hormone/steroid

- Nausea

$$
6(5)
$$

$40(31)$

$28(22)$

$17(13)$

- Nutrition supplement

- Insomnia

- Ventolin

- Insulin

- Antibiotics

- Blood pressure

- Opioid block

- Constipation

- PPI§

- Osteoporosis

- Incontinence

- Gout

- Eyes

- Epilepsy

- Blood

- Parkinson's Disease

- Allergies

Cumulative medications | |
$47(37)$

27 (21)

41 (32)

19 (15)

38 (30)

72 (56)

4 (3)

4 (3)

44 (34)

14 (11)

9 (7)

12 (10)

15 (12)

18 (14)

51 (40)

6 (5)

$2(2)$

$6(4,7)$

$41(62)$
$4(6)$
$12(18)$
$22(33)$
$19(29)$
$12(18)$
$6(9)$
$17(26)$
$9(14)$
$17(26)$
$14(21)$
$16(24)$
$37(56)$
$1(2)$
$18(27)$
$16(24)$
$8(12)$
$6(9)$
$5(8)$
$9(14)$
$10(15)$
$26(39)$
$3(5)$
$1(2)$

\begin{tabular}{|c|c|}
\hline 47 (76) & 0.095 \\
\hline $2(3)$ & 0.448 \\
\hline 11 (18) & 0.948 \\
\hline $26(42)$ & 0.315 \\
\hline $21(34)$ & 0.535 \\
\hline $16(26)$ & 0.297 \\
\hline 11 (18) & 0.149 \\
\hline $30(48)$ & 0.008 \\
\hline $18(29)$ & 0.033 \\
\hline 24 (39) & 0.117 \\
\hline $5(8)$ & 0.037 \\
\hline $22(36)$ & 0.164 \\
\hline 35 (57) & 0.964 \\
\hline $3(5)$ & 0.280 \\
\hline $22(36)$ & 0.317 \\
\hline $28(45)$ & 0.013 \\
\hline $6(10)$ & 0.658 \\
\hline $3(5)$ & 0.347 \\
\hline $7(11)$ & 0.471 \\
\hline $6(10)$ & 0.486 \\
\hline $8(13)$ & 0.715 \\
\hline $25(40)$ & 0.915 \\
\hline $3(5)$ & 0.937 \\
\hline $1(2)$ & 0.964 \\
\hline $6(5,7.25)$ & 0.02 \\
\hline
\end{tabular}

$6(3,6.25)$
* Comorbidity data in the medical record was unavailable for $n=4$ participants; + Comparison of well-nourished (SGA rating A) and malnourished (SGA rating B or C) groups; ₹ Number of comorbidities experienced by a single participant; data presented mean (SD); § PPI = proton pump inhibitor; । I Number of medication classes taken by a single participant; data presented median (IQR) 
Table 4

Health-related outcomes of the 2014 sample according to well-nourished or malnourished

\begin{tabular}{|c|c|c|c|c|}
\hline Outcomes & All participants $(n=128)$ & Well-nourished $(n=66)$ & Malnourished $(n=62)$ & P-value* \\
\hline Length of stay, days & $12(6,22)$ & $11(7-19)$ & $13(6-23)$ & $0.742+$ \\
\hline Hospital readmission & $76(60)$ & $37(56)$ & $39(63)$ & 0.431 \\
\hline UTI or respiratory infection & $68(53)$ & $35(53)$ & $33(53)$ & 0.982 \\
\hline Falls during admission & $38(30)$ & $20(30)$ & $18(29)$ & 0.875 \\
\hline Fractures on/during admission & $22(17)$ & $12(18)$ & $10(16)$ & 0.758 \\
\hline
\end{tabular}

Table 5

Health-related outcomes of the 2014 sample according to severely malnourished or not severely malnourished

\begin{tabular}{llll}
\hline Outcomes & No, mild, or moderate malnutrition $(\mathbf{n}=\mathbf{1 1 2})$ & Severe malnutrition $(\mathbf{n}=\mathbf{1 6})^{\text {p-value* }}$ \\
\hline Length of stay, days & $11.0(6.0,20.0)$ & $15.5(10.5,31.5)$ & $7(44)$ \\
Hospital readmission & $69(62)$ & $6(38)$ & $0.247+$ \\
In hospital mortality & $13(12)$ & $7(44)$ & 0.174 \\
UTI or respiratory infection & $61(55)$ & $7(44)$ & 0.006 \\
Falls during admission & $31(28)$ & $0(0)$ & 0.422 \\
Falls during subsequent admissions & $25(22)$ & $0(0)$ & 0.188 \\
Pressure wounds & $8(7)$ & $4(25)$ & 0.270 \\
Fractures on/during admission & $18(16)$ & 0.376 \\
\hline
\end{tabular}

Data expressed as median (IQR) or $\mathrm{n}(\%)$; * Comparison of no severe malnutrition (SGA rating A or B) and severely malnourished (SGA rating C) groups; + Test

performed on $\log 10$ normalised data

participants $(16 \%)(\mathrm{p}=0.028)$; where prevalence and total number of other comorbidities between groups were similar. Both groups had a median of six classes of medications prescribed during hospitalisation; where the malnourished group had a higher range (IQR 5, 7.25) than the well-nourished group (IQR 3, 6.25) ( $p=0.024)$. Malnourished participants were also more likely to be prescribed nutritional supplements $(\mathrm{p}=0.008)$ and protonpump inhibitors $(\mathrm{p}=0.013)$, and less likely to be prescribed medication for insomnia $(\mathrm{p}=0.037)$.

\section{Point-prevalence of malnutrition from 2012 to 2019}

Across the three time-points, malnutrition according to the SGA shows a peak in the 2014 cohort (48\%), which was significantly higher than in 2019 (28\%), but not 2012 (39\%) (Table 2). The prevalence of participants assessed as severely malnourished (SGA rating C) decreased over time from 15\% in 2012, 13\% in 2014, to 2\% in 2019 $(\mathrm{p}=0.005)$; whereas the prevalence of well-nourished fluctuated from $61 \%$ in $2012,52 \%$ in 2014 , to $72 \%$ in 2019 ( $\mathrm{p}=0.005)$. In a model adjusted for age and sex, regression analyses found that only age was a predictor of malnutrition, where each year of life increased the odds of malnutrition by $2 \%$ (OR: 1.020 [95\%CI: 1.003, 1.036] $\mathrm{p}=0.018$ ) but explained only $5 \%$ of variation in the model.

'Effect size' and 'chance of impact' was reported for comparisons between year groups for nutrition status to determine the magnitude of the difference between groups and whether or not the outcome was likely to have an actual impact. The pairwise comparison for nutrition status between 2014 and 2019 had a medium effect and 93\% chance of impact. For age comparison, 2014 versus 2019 had a medium effect and 89\% chance of impact. The 2012 versus 2019 comparison has a small effect size and a $35 \%$ chance of impact.

\section{Association of malnutrition with health-related outcomes}

In the 2014 sample, there were five extreme outliers for LOS that were removed. The average LOS was 12 (IQR: 6,22 ) days and $60 \%$ of participants were readmitted to hospital within 3-months. Malnourished participants (SGA rating $\mathrm{B}$ or $\mathrm{C}$ ) had a higher rate in-hospital mortality but this did not quite reach significace $(21 \%$ versus 9\%; $\mathrm{p}=0.059)$; groups did not differ on other 
outcomes (Table 4). Malnutrition was not a significant predictor of any health-related outcome in adjusted regression models.

Severely malnourished participants (SGA C) had a higher rate in-hospital mortality (38\% versus $12 \%$; $\mathrm{p}=0.006)$, and a lower rate of falls during subsequent admissions ( $0 \%$ versus $22 \%$; $\mathrm{p}=0.035)$; but groups did not differ on other outcomes (Table 5). Severe malnutrition increased the risk of in-hospital mortality by $457 \%$ (OR: 4.57 [95\%CI: 1.42, 14.66], $\mathrm{p}=0.011$ ); but in a model adjusted for age, cancer diagnosis, and prescription of nutrition supplements (confounders which met assumptions), this was reduced to $347 \%$ with a trend for significance [OR: 3.47 (95\%CI: 0.94, 12.78] $p=0.061)$. Severe malnutrition was not a predictor of other healthrelated outcomes in adjusted or unadjusted models.

\section{Discussion}

This study has reported a consistently high prevalence of malnutrition in three rural hospitals in northern NSW from 2012 to 2019; however, in 2019 the prevalence was $11 \%$ and $20 \%$ lower than in the previous samples, and the prevalence of severe malnutrition was very low at 2\%. The 2014 sample reported the highest prevalence of malnutrition in any Australian hospital (45\%); which exceeds the rate reported in three remote Australian hospitals (42\%) (19-21). The lowest prevalence reported in 2019 aligns with prevalence rates in Australian metropolitan hospitals. As nutrition status comparison between the 2014 and 2019 cohorts had a calculated medium effect size and high impact value, there is high confidence in the measured prevalence rates. Relevant for the Australian health care system, the SGA tool used to determine prevalence is synonymous with the International Classification for Diseases, 10th revision, Australian Modification (ICD-10-AM) classification of protein-energy malnutrition (22), and therefore directly linked to case-mix funding.

The differing rates in malnutrition prevalence over time is partially explained by the age of participants; however, the impact of age cannot account for the changes in prevalence alone. The lower rate of malnutrition in 2019 compared 2014 may also be due to variations in the sampled wards and hospital sites. The 2019 sample did not include a 34-bed rehabilitation ward; a setting which has previously been reported to have a high prevalence of malnutrition at 53\% (23). There are also likely causes of variation in the prevalence of malnutrition over time which were not captured by this study, including demographics such as socioeconomic status or ethnicity, inter-rater variability of SGA assessment, or changes in hospital policies and priorities to address malnutrition. Interestingly, the rate of malnutrition did not vary according to sex. A recent meta-analysis of worldwide data found that females had a $45 \%$ increased risk of malnutrition (OR: 1.45 [95\%CI:
$1.27,1.66] \mathrm{p}<0.00001)$ in the community setting, which included post-hospital samples.

It has been well established that malnutrition increases the risk of poor health outcomes in the hospital setting, $(21,24,25)$. This study confirms that malnourished participants had higher rates of in-hospital mortality; however, only severe malnutrition was a predictor of this outcome. The clinical importance of this is still relevant despite the rate of severe malnutrition being reduced to only $2 \%$ in 2019 , as there is a possibility of inter-rater variability in application of the SGA. To confirm if the risk of malnutrition-related in-hospital mortality has been eradicated with the decrease in severe malnutrition rates, health outcomes of the 2019 would need to be examined. A high rate of false positives in the 2014 sample would also explain why many participant characteristics usually associated with malnutrition were not significant predictors in multivariable models. Although severe malnutrition appeared to have a lower rate of falls in subsequent hospital admissions compared to better nourished participants, this is explained by very low rates of hospital readmission reflecting the high rate of in-hospital mortality in this group.

Of clinical significance, less than $50 \%$ of malnourished participants in the 2014 sample were provided with nutritional supplementation, and non-supplementation was a predictor of poor health-related outcomes in the adjusted models, including the high rate of hospital readmission within 3-months. Additionally, prescription of proton-pump inhibiters, which inhibit nutrient digestion, was higher in malnourished participants. Overall, the 2014 sample had a high rate of polypharmacy, a risk factor for malnutrition (26). Although comorbidities were highly prevalent, the only disease which was higher in the malnourished participants was cancer. Whilst malnutrition in cancer is known to highly prevalent (27); previous studies have identified that patients with other hypermetabolic conditions such as hepatic, cardiovascular, and gastrointestinal disease, depression, and dementia also have increased risk of malnutrition $(28,29)$.

\section{Limitations}

As discussed above, this study is limited by potential poor inter-rater reliability for the SGA assessment between samples, and not including further demographic data to explore variation in the multivariable models. In addition, as the SGA assessments were implemented cross-sectionally, the ratio of pre-existing malnutrition (i.e. admitted to hospital with malnutrition) to hospital acquired malnutrition within the reported prevalence is unclear. Finally, the 2014 sample may have been underpowered to detect differences in health-related outcomes, particularly risk of in-hospital mortality $(\mathrm{p}=0.059)$. 


\section{Conclusion}

Although the prevalence of malnutrition decreased over time, the rate of malnutrition in the sampled rural hospitals was consistently high; and is associated with increased risk of in-hospital mortality. Research should continue to monitor the rate of malnutrition in acute hospitals in rural areas to evaluate the impact of health service policies and procedures to address this problem.

\section{Key Question Summary}

What is known about the topic? Malnutrition is highly prevalent in the acute hospital setting in Australia at $30-40 \%$ and up to $71 \%$ in older adults (1). The Australian health system faces unique challenges related to high proportions of older adults living in geographically rural and remote areas. The rates and health-related complications of malnutrition in rural and remote Australian hospitals is unexplored.

What does this paper add? This study reported the highest ever recorded prevalence of malnutrition in Australia, at $48 \%$ in 2014; which was associated with increased risk of death. However, in 2019 the prevalence has reduced to $28 \%$, and severe malnutrition was almost eradicated (down to $2 \%$ ).

What are the implications for practitioners? Although the prevalence of malnutrition decreased over time, the rate of malnutrition in the sampled rural hospitals was consistently high; and was associated with increased risk of in-hospital mortality. Less than $50 \%$ of malnourished participants were provided with nutritional supplementation, and non-supplementation was a predictor of poor health-related outcomes in adjusted models, including in-hospital mortality.

Acknowledgements: A great thank you to Bond University statistician Evelyn Rathbone and the staff at Tweed, Murwillumbah, and Byron Bay Hospitals for their support of this study.

Conflicts of Interest: MB is the nutrition and dietetics department manager for the sampled sites and oversaw the implementation of all three cross-sectional studies. MB was not involved in the analysis of results. All other authors declare no existing or potential conflict of interest.

Funding sources: This research did not receive any specific grant from funding agencies in the public, commercial, or not-for-profit sectors.

Author contributions: All authors contributed to the conception of the study. EL and $\mathrm{MB}$ contributed to data collection. EL and SM contributed to data analysis. EL drafted the manuscript, and all authors contributed to manuscript revision. All authors approve the final version of the manuscript.

\section{References}

1. Banks M, Ash S, Bauer J, Gaskill D. Prevalence of malnutrition in adults in Queensland public hospitals and residential aged care facilities. Nutrition \& Dietetics. 2007;64(3):172-8.

2. Camilo ME. Disease-related Malnutrition: An Evidence-based Approach to Treatment. Clinical Nutrition. 2003;22(6):585.

3. Marshall S, Bauer J, Isenring E. The consequences of malnutrition following discharge from rehabilitation to the community: a systematic review of current evidence in older adults. J Hum Nutr Diet. 2014;27(2):133-41.

4. Marshall S. Protein-energy malnutrition in the rehabilitation setting: Evidence to improve identification. Maturitas. 2016;86:77-85.

5. Crichton M, Craven D, Mackay H, Marx W, de van der Schueren M, Marshall
S. A systematic review, meta-analysis and meta-regression of the prevalence of protein-energy malnutrition: associations with geographical region and sex. Age Ageing. 2018;48(1):38-48.

6. Agarwal E, Ferguson M, Banks M, Batterham M, Bauer J, Capra S, et al Malnutrition and poor food intake are associated with prolonged hospital stay, frequent readmissions, and greater in-hospital mortality: results from the Nutrition Care Day Survey 2010. Clin Nutr. 2013;32(5):737-45.

7. Agarwal E, Marshall S, Miller M, Isenring E. Optimising nutrition in residential aged care: a narrative review. Maturitas. 2016;92:70-8.

8. Pirlich M, Schütz T, Norman K, Gastell S, Lübke HJ, Bischoff SC, et al. The German hospital malnutrition study. Clin Nutr. 2006;25(4):563-72.

9. Marshall S. Why is the skeleton still in the hospital closet? A look at the complex aetiology of malnutrition and its implications for the nutrition care team. J Nutr Health Aging. 2018;22:26-9.

10. Curtis LJ, Bernier P, Jeejeebhoy K, Allard J, Duerksen D, Gramlich L, et al Costs of hospital malnutrition. Clin Nutr. 2017;36(5):1391-6.

11. Marshall S, Agarwal E. Comparing characteristics of malnutrition, starvation, sarcopenia and cachexia in older adults. Handbook of famine, starvation, and nutrient deprivation: from biology to policy. 2017:1-23.

12. Caring for Older Australians, Volume 1. Canberra: Productivity Commission; 2011.

13. Living Longer. Living Better. Aged Care Reform Package. Canberra: Australian Government Department of Health and Ageing; 2012.

14. Caring for Older Australians, Volume 2. Canberra: Productivity Commission 2011

15. Von Elm E, Altman DG, Egger M, Pocock SJ, Gøtzsche PC, Vandenbroucke JP, et al. The Strengthening the Reporting of Observational Studies in Epidemiology (STROBE) statement: guidelines for reporting observational studies. Prev Med. 2007;45(4):247-51.

16. Agarwal E, Ferguson M, Banks M, Vivanti A, Batterham M, Bauer J, et al. Malnutrition, poor food intake, and adverse healthcare outcomes in non-critically ill obese acute care hospital patients. Clinical Nutrition. 2019;38(2):759-66

17. Detsky AS, McLaughlin JR, Paker JP, Johnston N, Wittaker S, Mendelson RA, et al. What is subjective global assessment of nutritional status. JPEN. 1987;11:8-11.

18. Marshall S, Craven D, Kelly J, Isenring E. A systematic review and metaanalysis of the criterion validity of nutrition assessment tools for diagnosing protein-energy malnutrition in the older community setting (the MACRo Study). Clin Nutr. 2018;37(6A):1902-12

19. Morris NF, Stewart S, Riley MD, Maguire GP. The burden and nature of malnutrition among patients in regional hospital settings: A cross-sectional survey. Clinical Nutrition ESPEN. 2018;23:1-9.

20. Allard JP, Keller H, Jeejeebhoy KN, Laporte M, Duerksen DR, Gramlich L, et al. Decline in nutritional status is associated with prolonged length of stay in hospitalized patients admitted for 7 days or more: A prospective cohor study. Clinical Nutrition. 2016;35(1):144-52.

21. Agarwal E, Ferguson M, Banks M, Batterham M, Bauer J, Capra S, et al. Malnutrition and poor food intake are associated with prolonged hospital stay, frequent readmissions, and greater in-hospital mortality: results from the Nutrition Care Day Survey 2010. Clin Nutr. 2013;32(5):737-45.

22. Australian coding standards for I.C.D.-10-AM. Sydney: National Centre for Classification in Health; 2008.

23. Marshall S, Young A, Bauer J, Isenring E. Malnourished older adults admitted to rehabilitation in rural New South Wales remain malnourished throughout rehabilitation and once discharged back to the community: a prospective cohort study Journal of Aging Research and Clinical Practice. 2015;4(4):197204.

24. Allard JP, Keller H, Jeejeebhoy KN, Laporte M, Duerksen DR, Gramlich L, et al. Malnutrition at Hospital Admission-Contributors and Effect on Length of Stay: A Prospective Cohort Study From the Canadian Malnutrition Task Force. JPEN J Parenter Enteral Nutr. 2016:40(4):487-97.

25. Sanson G, Bertocchi L, Dal Bo E, Di Pasquale CL, Zanetti M. Identifying reliable predictors of protein-energy malnutrition in hospitalized frail older adults: A prospective longitudinal study. Int J Nurs Stud. 2018;82:40-8.

26. Nutrition screening as easy as mna. A guide to completing the Mini Nutritional Assessment (MNA). Swizterland Nestle Nutrition Institute.

27. Segura A, Pardo J, Jara C, Zugazabeitia L, Carulla J, de las Penas R, et al. An epidemiological evaluation of the prevalence of malnutrition in Spanish patients with locally advanced or metastatic cancer. Clin Nutr. 2005;24(5):80114.

28. Konturek PC, Herrmann HJ, Schink K, Neurath MF, Zopf Y. Malnutrition in Hospitals: It Was, Is Now, and Must Not Remain a Problem! Medical science monitor : international medical journal of experimental and clinical research 2015;21:2969.

29. Bonetti L, Terzoni S, Lusignani M, Negri M, Froldi M, Destrebecq A. Prevalence of malnutrition among older people in medical and surgical wards in hospital and quality of nutritional care: A multicenter, crosssectional study. Journal of clinical nursing. 2017;26(23-24):5082-92.

29. Faul, F., Erdfelder, E., Buchner, A., \& Lang, A.-G. (2009). Statistical power analyses using $G^{*}$ Power 3.1: Tests for correlation and regression analyses. Behavior Research Methods, 41, 1149-1160. 